\title{
A New Clustering Algorithm for Live Road Surveillance on Highways based on DBSCAN and Fuzzy Logic
}

\author{
Hasanain Alabbas ${ }^{1}$, Árpád Huszák ${ }^{2}$ \\ Department of Networked Systems and Services \\ Budapest University of Technology and Economics \\ H-1117 Budapest, Hungary ${ }^{1,2}$ \\ Al-Qasim Green University, Babylon, Iraq ${ }^{1}$
}

\begin{abstract}
Video streaming over Vehicular Ad Hoc Networks is a promising technique (VANETs), and it has gained great importance in the last few years. The highly dynamic topology of VANETs makes high-quality video streaming very challenging. In order to provide the most useful camera views to the vehicles, clustering and cluster head selection techniques are used. Too frequent camera view changes can be annoying; therefore, we propose a new stable clustering algorithm to ensure a stable live road surveillance service without interruptions for vehicles that do not have enough vision area. In the proposed solution, we integrated Density-Based Spatial Clustering of Applications with Noise (DBSCAN) with Fuzzy Logic Control (FLC). DBSCAN is used to form the clusters, while FLC is used to find the best cluster head for the cluster. Different parameters are utilized like density parameters for DBSCAN, and relative speed, acceleration, leadership degree and vision area for fuzzy logic. Our proposed algorithm showed better results in terms of cluster lifetime and vehicle status change. Our proposed algorithm has been compared with another clustering scheme to prove the effectiveness of our proposed algorithm.
\end{abstract}

Keywords-Vehicular ad hoc networks (VANETs); V2V; intelligent transportation systems; clustering algorithms; road surveillance; DBSCAN algorithm; fuzzy logic control

\section{INTRODUCTION}

Recently, Vehicular Ad Hoc Network (VANET) is deemed to be one of the most vital subjects which have gained noteworthy research consideration [1]. It is part of Intelligent Transportation Systems (ITS), which is intended to provide reliable communication between vehicles and fixed roadside units and among vehicles themselves by enabling them to make contact with each other directly [2]. VANET applications can be classified into safety, traffic efficiency, and luxury applications [3].

The communication entities, Road Side Unit (RSU) and On-Board Unit (OBU) form the backbone of VANET infrastructure. OBU is a communication device fixed in the vehicle, while RSU is a fixed unit distributed on the side of the roads or near the traffic signals [4]. In general, two connection types can be set up by this communication equipment, Vehicle-to-Vehicle (V2V), which enables the vehicular nodes to contact each other directly and Vehicle-toInfrastructure (V2I), in which vehicles can communicate with
RSUs [5]. The standard 802.11p applied in VANETs is a branch of the standard IEEE 802.11, which represents a suitable solution for vehicular communications [6]. It has been mainly designed to provide a short-to-medium transmission range for high-speed vehicles up to (300 m - $1000 \mathrm{~m}$ ) and to meet every V2V and V2I application [7].

VANETs inherit the main characteristics of Mobile Ad Hoc Networks (MANETs), in which the vehicles play the role of mobile nodes supplied with wireless communications [8]. Many applications are designed for safety and non-safety purposes, and one of these applications is the video streaming, which is now the focus of research community attention because it enriches the drivers and passengers with valuable information in comparison with textual messages [9]-[11]. Many challenges face video streaming service, which is considered highly-bandwidth-demanded like network congestion and the highly dynamic topology of VANETs. The network congestion happens if there are many vehicles that broadcast this service at the same time [12]. These factors have a significant impact on video streaming services.

Clustering strategies are considered one of the most effective solutions that were applied in VANETs to enhance the performance of the system, increase the scalability of the network and provide good management by grouping the vehicles into groups depending on some metrics [13].

Although VANET is a sub-class of MANETs, the conventional clustering strategies designated for MANET is unworkable to VANET directly because VANET has different challenges. The nodes in VANET can be connected or disconnected to the network very quickly, causing a highly dynamic topology and a frequently disconnected network. They are also constrained by the route of the roads and traffic regulation [15]. These parameters have a massive influence on the communication stability. A broad range of clustering algorithms was presented for VANET, and many issues are addressed by them. One of the problems, which is considered essential for clustering design in VANETs, is how to improve cluster stability [16]-[18]. In general, the VANET clustering algorithm divides the vehicular nodes into virtual sets named clusters. Each group elects a cluster head $(\mathrm{CH})$ according to some rules set, while the other nodes in the group join the cluster as cluster members (CM). The $\mathrm{CH}$ is responsible for 
cluster maintenance and coordinating the transmission among CMs in the same way as an infrastructure wireless access point.

In this paper, we propose a new algorithm aiming to achieve stable clusters and find a suitable $\mathrm{CH}$ for vehicles that tend to get the road conditions via video surveillance service. DBSCAN technique is used to configure the clusters, while Fuzzy Logic Control (FLC) is used to select the best $\mathrm{CH}$. The elected $\mathrm{CH}$ will be responsible for providing video surveillance on the conditions of the road to all CMs in the same cluster, depending on the on-board camera substantiated inside the vehicle. Our proposed scheme has been compared with the Effective-Vision-Area-Based Clustering Algorithm with the Adaptive Video-Streaming Technique (EVAC-AV) algorithm and showed an effective result in increasing the cluster lifetime.

This paper is organized as follows. Section II presents the literature review. Section III describes the proposed clustering approach. In Section IV, the simulation environment and the methodology is shown. The performance evaluation and results are introduced in Section V. Finally; Section VI concludes the paper.

\section{RELATED WORK}

The clustering mechanism is an effective technique, which is used to streamline some critical functions like media access management, quality of service achievement, and bandwidth allocation, etc. In general, the nodes in the clustering algorithms have three states: $\mathrm{CH}$ state, normal state (NS), and CM state. These terms may vary in some articles, but they have the same notions. $\mathrm{CH}$ is the focal point of the cluster, which is elected to coordinate the cluster, while NS represents the state of a node that does not belong to any cluster. When it joins a cluster, it becomes a CM. Fig. 1 shows the topology of three clusters, in which each cluster elects a single $\mathrm{CH}$. It clarifies how the different nodes are formed and grouped.

Due to the significance of the issues that clustering addresses, many clustering methods have been proposed lately in the context of VANETs. Most of them aim to achieve network constancy.

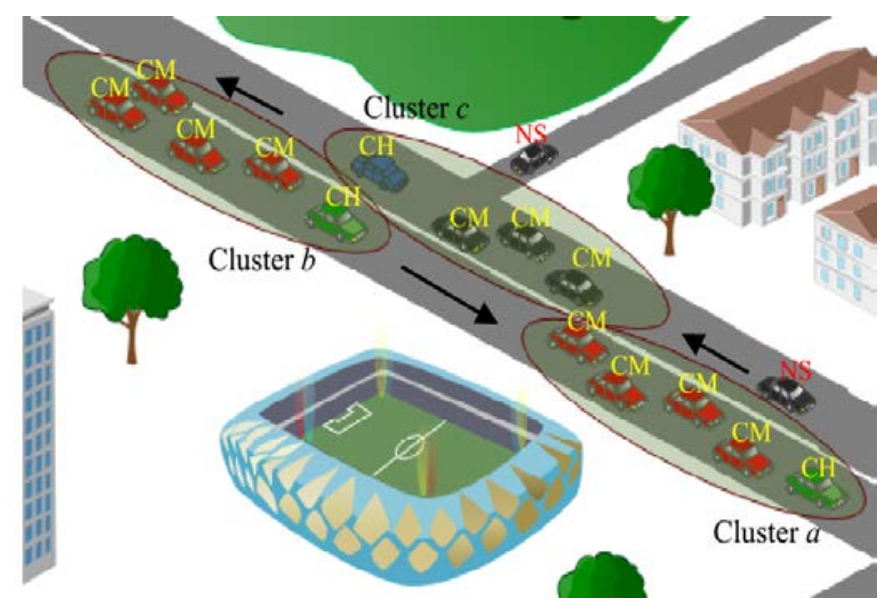

Fig. 1. An Example of a Cluster Network Topology.
Amjad Mehmood and et al. [19] have employed the flow of traffic knowledge in addition to using several metrics, like the degree of connectivity, the node position, direction, and speed variation to form stable clusters. The naïve Bayesian probabilistic estimation technique is used to enhance cluster stability and increase the $\mathrm{CH}$ lifetime. The proposed technique was compared with other algorithms and showed improvements in cluster and $\mathrm{CH}$ lifetime. Regardless of the efficiency of the ANTSC algorithm in selecting the $\mathrm{CH}$ and increasing the cluster lifetime, it is used for a particular scenario, so it was unclear whether it could be used in different scenarios. Moreover, the naïve Bayesian network probabilistic estimation requires real datasets for each zone, which makes it inapplicable in case lack of dataset.

The authors in [20], proposed a new clustering algorithm to select the most suitable $\mathrm{CH}$ based on FLC. A blend of several metrics was considered as inputs of the proposed cluster head selection algorithm, such as speed, distance, acceleration, and direction. The results showed that developed fuzzy logic (FL) based Cluster Head Selection Algorithm (CHSA) has increased the stability of $\mathrm{CH}$ and improved generally the performance in various scenarios in VANETs.

The Fuzzy-Based Cluster-Management System (FBCMS) has been proposed in [21]. Two models of this system have been created of this system, where each model has different parameters to select the most appropriate cluster head. The first model utilizes three parameters, which are the group speed, relative acceleration, and security as inputs of fuzzy logic, while the second model uses four metrics. Three of them are the same as the first model in addition to the degree of connectivity as the fourth parameter. However, using the location of vehicles in relation to a fixed RSU as one of the parameters to determine the $\mathrm{CH}$ in a highly dynamic environment like VANET could have a negative impact on the stability of clusters and may lead to frequent network disconnection, especially on highways.

The authors of [22] proposed a novel clustering scheme, which depends on the average speed of vehicles and standard deviation to increase the cluster lifetime. Two clustering patterns have been introduced which rely mainly on the principle of the normal (Gaussian) distribution and the relative speed. The calculated residence time of vehicular nodes in a cluster is used as a stability criterion. The first pattern represented a very high stable cluster in which the vehicles having speeds within the range of mean and standard deviation are used to configure this cluster (i.e., only $68 \%$ of the vehicles permitted to form this cluster). The election of the cluster head is carried out from the vehicles having speeds close to the average of cluster speed. The second pattern aims to group about $95 \%$ of the vehicles by selecting only the vehicles having speeds with a deviation lower than the double of the average standard deviations $(\sigma \leq 2 \bar{\sigma})$ in one cluster. The analytical analysis showed that the second pattern has less stability than the first one. These two metrics (average speed and standard deviation) alone are not enough to establish stable clusters and select the optimal cluster heads for them, particularly as many parameters should have been taken like acceleration and position. 
About video streaming and live road surveillance, the EVAC-AV [23] has been proposed as a solution for this kind of clustering. The cluster is initiated when a vehicle disseminates a request to join a cluster for having a live road surveillance service, so the vehicles which are ahead of it will be triggered to calculate their vision area. If their vision area is larger than a predefined vision area threshold, they will be deliberated as candidate CHs. Using the largest vision area as a single parameter to determine the best cluster head is not enough to create a stable cluster especially since the video streaming service is the most affected by the changes and reclustering furthermore, the other algorithms which aim to provide stable clusters depend mainly on RSUs as a key parameter which makes it difficult to apply these algorithms in highways environments lacking to V2I technologies, therefore, this paper proposes a new stable V2V clustering algorithm entitled "A New Clustering Algorithm for Live Road Surveillance on Highways Based on DBSCAN and Fuzzy Logic". This algorithm aims to create stable clusters based on the density of vehicles on the street by using DBSCAN to form clusters and select the optimal $\mathrm{CH}$ based on FLC. DBSCAN ensures constructing the clusters without having to rely on RSUs as other algorithms do while FLC is used to select the best cluster head.

\section{PROPOSED ALGORITHM}

This paper presents a new algorithm that has the ability to detect and form a cluster automatically when the density of vehicles increases as well as selects the optimal $\mathrm{CH}$. The strength of our algorithm is derived from the integration of the DBSCAN algorithm with fuzzy logic control. In our assumption, all vehicles are fitted with OBUs to be able to handle the IEEE802.11p as a Dedicated Short Range Communications (DSRC) system. Each vehicle broadcasts its information by sending Cooperative Awareness Message (CAM), which is a single hope broadcast communication. This CAM message is sent periodically at regular time intervals called $T_{\text {update }}$. Based on these messages, each vehicle will sense its current neighboring vehicles and update its Neighbor Table through exchanging the speed, position, and direction. Our proposed algorithm aims to provide a stable density-based clustering technique on a highway that consists of two phases cluster configuration phase and cluster head selection phase. Fig. 2 depicts our proposed algorithm.

\section{A. Overview of DBSCAN}

Clustering represents the most commonly used and more powerful unsupervised learning mechanism in machine learning. It is a useful tool that aims to classify the input data into sets depending on some similarity calculations. These algorithms are categorized into groups like partitional algorithms, density-based algorithms, hierarchical algorithms, etc. [24]. Among them, DBSCAN has been selected in our proposed algorithm because it has many features that make it more suitable than other clustering techniques. DBSCAN is an effective density-based clustering algorithm for spatial data systems due to its ability to discover clusters with arbitrary shapes in one scan, not like, for example, K-mean, which needs many iterations to find out the clusters. It is characterized by its capability to detect outliers as well as it does not need to predetermine the number of clusters. In DBSCAN, the distance of two points is determined by a distance metric, such as the Euclidean distance. However, there are two parameters in DBSCAN which are required to be specified, Epsilon $(\varepsilon)$ and Minimum Points (MinPts). $\varepsilon$ represents the maximum distance between two points, which means that if the distance between two points is lower or equal $\varepsilon$, these points are considered neighbors. MinPts represents the minimum number of points counted neighbors for that point. It is used to identify if the point is a core point, border point, or noise point [25].

\section{B. Vehicle Vision Area}

Vision area plays an essential role in defining the cluster topology and electing the $\mathrm{CH}$ because the $\mathrm{CH}$ is responsible for providing live video surveillance to all vehicles located behind it that do not have enough vision area. No vehicle can be nominated to be a $\mathrm{CH}$ if it does not have a sufficient vision area. Therefore, the vehicles will be classified into two classes: (i) vehicles that have vision area $\left(V_{\text {vision }}\right)$, which can be potential CHs; (ii) vehicles that do not have vision area $V_{\text {no- }}$ vision, which can be possible CMs. We assume each vehicle has a camera mounted on the vehicle dashboard to capture live road conditions. The Distance Threshold $\left(D_{t h}\right)$ is used to define the vision area. We can say any vehicle is a $V_{\text {vision }}$ if the distance between it and the adjacent front vehicle on the same lane is less than the $D_{t h}$, but if the distance is less than $D_{t h}$, it is considered a $V_{\text {no-vision. }}$. It is worth noting that the $D_{t h}$ value is the same as the value of $\varepsilon$ parameter used in the DBSCAN algorithm, which represents the safety distance that gives the driver the sufficient time for appropriate decision in case if he decides to overtake or in case of any emergency like a sudden incident.

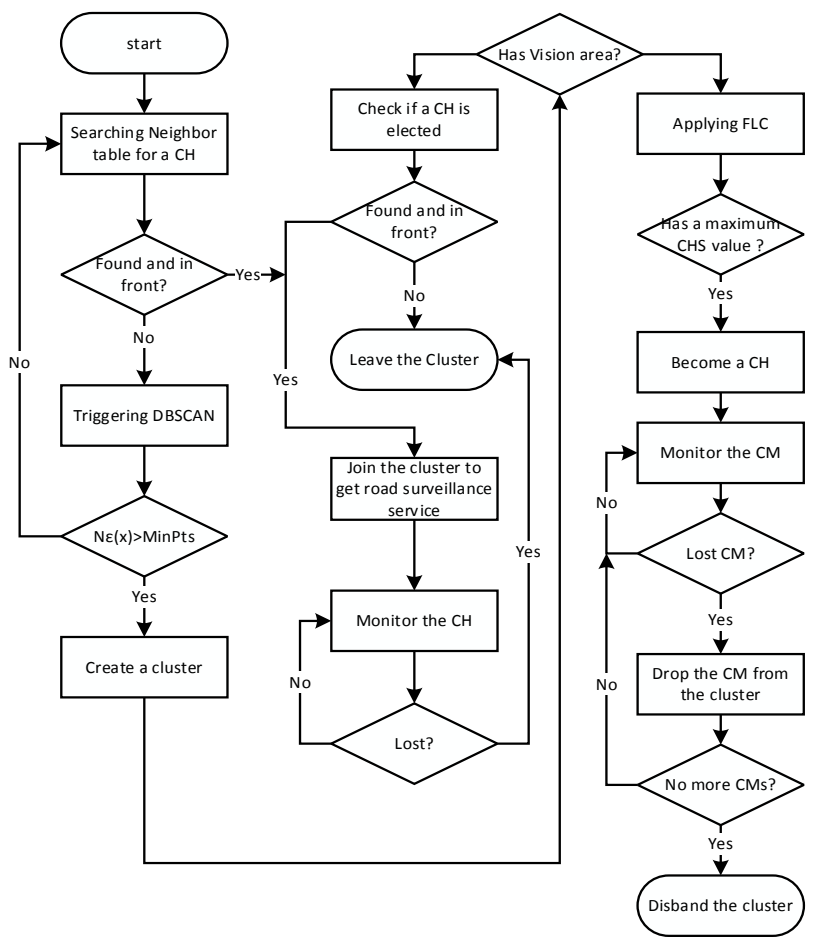

Fig. 2. Flowchart of Our Proposed Algorithm. 


\section{Cluster Configuration}

At the beginning of clustering, each vehicle initializes its state to NS state, which means it does not belong to any cluster yet. Then, each vehicle shares its information by sending CAM message, which contains the speed, position, direction, and vision area. The Neighbor Table is updated periodically in a time interval called $T_{\text {update }}$. Each vehicle will filter the Neighbor Table and select only the vehicles in the same direction to prevent the vehicles on the reverse side or in a different street from shortly participating in the cluster. Referring to Fig. 2 DBSCAN will establish the cluster when it detects enough nearby vehicles close to each other based on $\varepsilon$ and MinPts. Let us suppose the vehicle $x$ moving on the highway does not have enough vision area. It will search in its Neighbor Table about a suitable $\mathrm{CH}$. The suitable $\mathrm{CH}$ should be in front of it and should have enough vision area to provide efficient live video streaming about road conditions. If vehicle $x$ does not find a suitable $\mathrm{CH}$, it will filter the Neighbor Table and only collects the positions of vehicles in an NS, which have the same direction to form a dataset and then trigger the DBSCAN algorithm.

In DBSCAN, the vehicles are adjacent to the vehicle $\mathrm{x}$, denoted by $N_{\varepsilon}(x)$ is defined by.

$N_{\varepsilon}(x)=\{y \in D \mid$ distance $(x, y) \leq \varepsilon\}$,

Where $y$ any vehicle in the dataset of vehicle $x$ and $D$ represents the DBSCAN dataset. Three types of nodes are defined in DBSCAN:

1) Core Node: The vehicle $x$ is considered a core node if $\left|N_{\varepsilon}(x)\right| \geq$ MinPts.

2) Border Node: The vehicle $x$ is considered a border node if $\left|N_{\varepsilon}(x)\right|<$ MinPts, but one of the $N_{\varepsilon}(x)$ is a core node.

3) Noise Node: The vehicle $(x)$ is considered a noise node if $\left|N_{\varepsilon}(x)\right|<$ MinPts and no one of $N_{\varepsilon}(x)$ is a core node.

After DBSCAN has found a core node, the remaining adjacent vehicular nodes are checked consecutively to identify the next core node. If another nearby node becomes a core point, the cluster domain is extended. DBSCAN continues this process until no more core points can be found. Fig. 3 shows how the cluster is established after being originated by vehicle $x$. All the vehicles are included in the group, except the vehicle considered as a noise node. It should be noted that the vehicle in the center of the blue circle is a core node, and the vehicle in the center of the yellow circle is a border node, while the vehicle in the center of the red circle is a noise node. In case of existing a suitable $\mathrm{CH}$, it will send a request to join the cluster and become a CM. In the case of more than one $\mathrm{CH}$ in front of it, it will select the closest one.

\section{Cluster Head Selection (CHS) Phase}

Cluster Head Selection (CHS) plays a significant role in cluster stability, which in turn represents one of the performance criteria in VANETs. The CHS process starts after cluster creation in which only $V_{\text {vision }}$ vehicles in the cluster will enter to CHS phase as Candidate Cluster Heads (CCHs). FLC is the technique used to find the most suitable $\mathrm{CH}$ in the cluster. Fuzzy logic is an effective multi-characteristic decision technique because of its ability to a trade-off between significance and precision. Three parameters are considered in the CHS phase: Cluster Speed (CS), Vehicle Acceleration (VAcc), and Leadership Degree (LD). CS is determined by calculating the average speed of the vehicles in the clusters. $L D$ is a value between 0 and 1 which shows if the $C C H$ is eligible to be a $\mathrm{CH}$ or not, where 0 means that the $\mathrm{CCH}$ is in the back of the cluster (all potential CMs are in front of it), which means it is not eligible to be a $\mathrm{CH}$ while 1 represents that the $\mathrm{CCH}$ is at the front of the cluster and has the highest degree of eligibility to be a $\mathrm{CH}$. The $L D$ metric is calculated for each $\mathrm{CCH}$ so

$$
L D(C C H)=\frac{\sum_{i=1}^{N} V_{B}}{\sum_{i=1}^{N} V_{n o-v i s i o n}}
$$

Where $N$ is the number of vehicular nodes in the cluster, $V_{B}$ is the number of $V_{\text {no-vision }}$ behind the $\mathrm{CCH}$ in the cluster. These three metrics are fuzzified using the fuzzy logic system. Fig. 4 shows our CHS System. As shown in this figure, three parameters (CS, VAcc, $L D)$ are considered as an input of the fuzzification system. The function of the fuzzification system is to convert the actual values of the input parameters into fuzzy sets by using membership functions. There are many types of membership functions.

In our CHS system, we have utilized triangular and trapezoidal membership functions, as shown in Fig. 5 because they are more efficient in real-time applications [26]. The term sets of $C S, V A c c$, and $L D$ are defined respectively as:

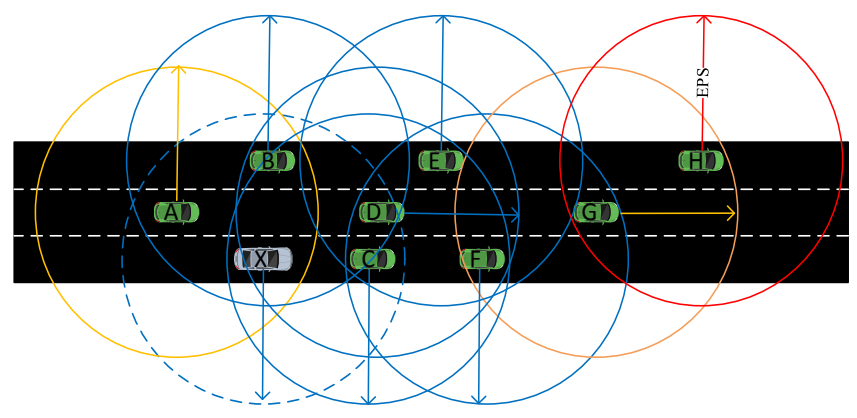

Fig. 3. DBSCAN Algorithm Configuration.

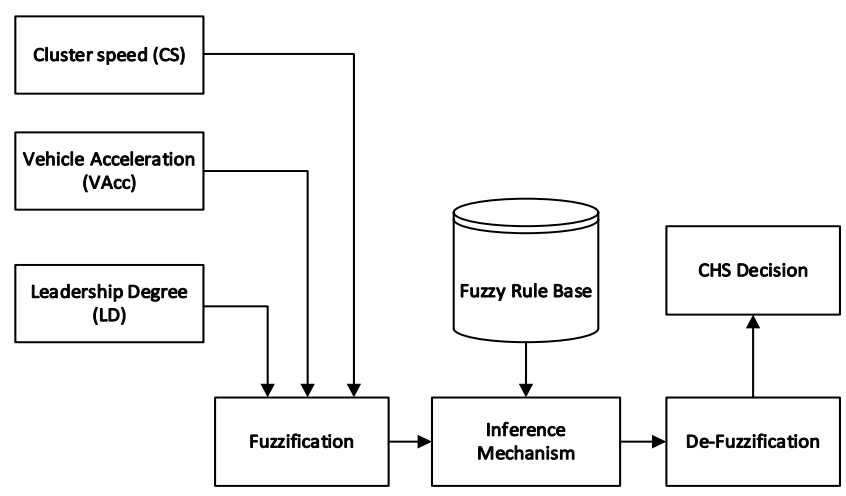

Fig. 4. CHS System. 
$C S=\{$ Low Speed, Normal Speed, High Speed $\}$

$=\{\mathrm{LS}, \mathrm{NS}, \mathrm{HS}\}$

$V A c c=\{$ Deceleration, Same Speed, Acceleration $\}$

$=\{$ Dec, SS, Acc $\}$

$L D=\{$ Low Degree, Normal Degree, High Degree $\}$

$=\{\mathrm{LD}, \mathrm{ND}, \mathrm{HD}\}$

$\mathrm{CHS}=\{$ Very low, Low, Medium, High, Very High $\}$

$=\{\mathrm{VL}, \mathrm{L}, \mathrm{M}, \mathrm{H}, \mathrm{VH}\}$

The Fuzzy Rule Base (FRB) is built based on these membership functions. Since we have three inputs, and each input has three values, then we need 27 rules to cover all possible inputs.

$$
X *=\frac{\sum_{j=1}^{27} x_{j}\left(\prod_{i=1}^{3} \mu\left(p_{i}\right)\right)}{\sum_{j=1}^{27}\left(\prod_{i=1}^{3} \mu\left(p_{i}\right)\right)}
$$
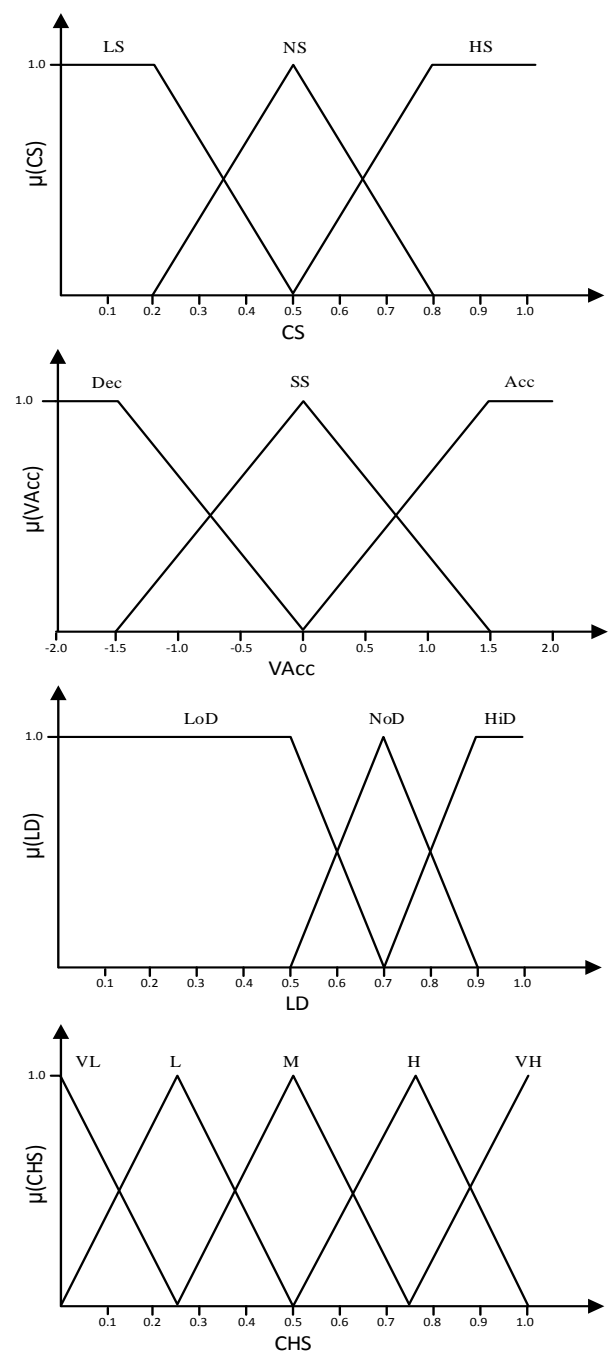

Fig. 5. Membership Functions of CHS System.
TABLE I. FUZZY RULE BASE OF CHS SYSTEM

\begin{tabular}{|c|c|c|c|c|}
\hline Rule No. & CS & VAcc & $L D$ & CHS \\
\hline 1 & $L S$ & Dec & $L o D$ & $V L$ \\
\hline 2 & $L S$ & Dec & NoD & $V L$ \\
\hline 3 & $L S$ & Dec & $H i D$ & $L$ \\
\hline 4 & $L S$ & SS & $L o D$ & $V L$ \\
\hline 5 & $L S$ & SS & $\mathrm{NoD}$ & $L$ \\
\hline 6 & $L S$ & SS & $\mathrm{HiD}$ & $M$ \\
\hline 7 & $L S$ & $A c c$ & $L o D$ & $V L$ \\
\hline 8 & $L S$ & $A c c$ & $N o D$ & $M$ \\
\hline 9 & $L S$ & $A c c$ & $\mathrm{HiD}$ & $H$ \\
\hline 10 & NS & Dec & $L o D$ & $V H$ \\
\hline 11 & NS & Dec & NoD & $L$ \\
\hline 12 & NS & Dec & $H i D$ & $M$ \\
\hline 13 & NS & SS & $L o D$ & $V L$ \\
\hline 14 & $N S$ & SS & $N o D$ & $H P$ \\
\hline 15 & $N S$ & SS & $H i D$ & $V H$ \\
\hline 16 & $N S$ & $A c c$ & $L o D$ & $V L$ \\
\hline 17 & NS & $A c c$ & $N o D$ & $H$ \\
\hline 18 & NS & $A c c$ & $H i D$ & $M$ \\
\hline 19 & $H S$ & Dec & $L o D$ & $V L$ \\
\hline 20 & $H S$ & Dec & $N o D$ & $H$ \\
\hline 21 & $H S$ & Dec & $H i D$ & $V H$ \\
\hline 22 & $H S$ & SS & $L o D$ & $V L$ \\
\hline 23 & $H S$ & SS & $\mathrm{NoD}$ & $M$ \\
\hline 24 & $H S$ & SS & $H i D$ & $H$ \\
\hline 25 & $H S$ & $A c c$ & $L o D$ & $V L$ \\
\hline 26 & $H S$ & $A c c$ & NoD & $L$ \\
\hline 27 & $H S$ & Acc & $\mathrm{HiD}$ & $V L$ \\
\hline
\end{tabular}

The CHS value $\left(X^{*}\right)$ is ranging between 0 and 1 . Table I shows the Fuzzy rule base of the CHS system. After determining the CHS value $\left(X^{*}\right)$ to all CCHs in the cluster, the $\mathrm{CCH}$ with maximum $\mathrm{CHS}$ value will declare itself as a $\mathrm{CH}$. All the vehicles behind the $\mathrm{CH}$ will send a Request message to get a video streaming service. When the $\mathrm{CH}$ receives the Request message, it sends Accept message back to them. After the vehicles receive Accept message, they will change their status from NS to CM. All the vehicles in front of the $\mathrm{CH}$ will leave the cluster.

\section{E. Disbanding the Cluster}

Referencing to Fig. 2, the $\mathrm{CH}$ will disband the cluster in two cases:

Case 1: When $\mathrm{CH}$ is blocked by other vehicles, so its vision area is less than the $D_{t h}$, it will disband the cluster.

Case 2: When the $\mathrm{CH}$ discovers that all its CMs have left the cluster, it will disband the cluster.

\section{F. Leaving the Cluster}

Two conditions can cause the CMs to leave the cluster. Each CM monitors its link to its CH every $T_{\text {update }}$. If it has not received the CAM message from the $\mathrm{CH}$ for two $T_{\text {update }}$, the vehicle's link to its $\mathrm{CH}$ fails, and in this case, it will leave the cluster and change its state from CM to NS. The CM also 
leaves the cluster when it overtakes the $\mathrm{CH}$ and becomes in front of it, so the video streaming service from the $\mathrm{CH}$ becomes useless. In this case, it will send a Leave message to its $\mathrm{CH}$.

\section{TOOLS AND METHODOLOGY}

Our proposed clustering algorithm has been evaluated using MATLAB R2017b, while the mobility of vehicles has been simulated by the Simulation of Urban Mobility (SUMO). Our algorithm is designed for highway, so a $5 \mathrm{~km}$ highway is modeled in SUMO. The road consists of six lanes, three lanes for each direction in which 50 vehicles moving in the same direction with different speeds were deployed. The speed of vehicles is ranging from $60-120 \mathrm{~km} / \mathrm{h}$. The standard $D_{\text {th }}$ is 50 $\mathrm{m}$, which is the distance approved as safety distance between the vehicles when the speed of vehicles is $100 \mathrm{~km} / \mathrm{h}$ [23]. In our work, we use different $D_{t h}$ to confirm the efficiency of our proposed algorithm. Concerning the DBSCAN parameters, we considered the number of the lane in the same direction represents the Minpts, and the safety distance represents the value of $\varepsilon$, so if the $D_{t h}$ is 50 , then $\varepsilon$ is $50 \mathrm{~m}$. The main parameters applied in the simulation are mentioned in Table II. MATLAB and SUMO blocks have been connected together by TraCI (Traffic Control Interface). TraCI creates a TCP connection to make a connection between MATLAB and SUMO. SUMO acts as a server (TraCI-Server) and MATLAB as a client (TraCI-Client).

TABLE II. SYSTEM SIMULATION PARAMETERS

\begin{tabular}{|l|l|}
\hline Parameters & Values \\
\hline Highway Distance & $5 \mathrm{Km}$ \\
\hline Simulation Time & $100 \mathrm{sec}$ \\
\hline Number of vehicles & 50 \\
\hline Distance threshold $\left(D_{t h}\right)$ & $(50,70) \mathrm{m}$ \\
\hline OBU transmitting range & $300 \mathrm{~m}$ \\
\hline Vehicles speed & $60,80,100,120 \mathrm{Km} / \mathrm{h}$ \\
\hline
\end{tabular}

\section{RESUlts}

The performance evaluation of the proposed clustering algorithm was done by comparing our results with EVAC-AV algorithm results. It should be noted that we chose EVAC-AV introduced in the related work section as a benchmark algorithm because it is the only clustering algorithm based on vision area estimations and use $\mathrm{V} 2 \mathrm{~V}$ mode. The rest of the other algorithms have been excluded from the comparison because they differ in terms of purpose, parameters, and calibration. Our aim is to improve the performance and increase the stability of $\mathrm{CHs}$ as well as decreasing the number of vehicles status change. The following two performance metrics were used:

- Average cluster lifetime: It is defined as how long each cluster will last continuously. A higher value of this measure denotes a better stability.

- Vehicles status change: Vehicle status change is defined as the number of status changes per vehicle during its lifetime.
Fig. 6 and Fig. 7 demonstrate the distribution of $\mathrm{CHs}$, CMs, and NSs during the simulation time when the $D_{t h}=50 \mathrm{~m}$ and $70 \mathrm{~m}$, respectively.

They show a high number of NSs in each time step. The reason for this is that these vehicles still in NS because they have enough vision area and do not need to join to any cluster as well as they are moving in an area with a low density of vehicles, so they are detected as noise nodes (NSs) during the forming the cluster by DBSCAN algorithm. This is considered a virtue because our proposed algorithm aims to form clusters and provides stable CHs for only the $V_{\text {no-vision }}$ that need video streaming to know the road conditions; therefore we classified the NSs into $\mathrm{NS}_{\text {no-vision }}$ and $\mathrm{NS}_{\text {vision }}$ to calculate the exact number of vehicles that do not have enough vision area in NS and do not find $\mathrm{CH}$. Fig. 8 shows the number of $\mathrm{NS}_{\text {vision }}$ and $\mathrm{NS}_{\text {no-vision }}$ during the simulation time. The results showed the percentage of remaining $\mathrm{NS}_{\text {no-vision }}$ is $2 \%$ during the simulation time when the $D_{t h}=50 \mathrm{~m}$ (Fig. 8 (a)) while it is up to $8 \%$ when $D_{\text {th }}=70 \mathrm{~m}$ (Fig. 8 (b)).

Our proposed algorithm is compared with EVAC-AV in the term of cluster lifetime and number of cluster. As shown in Fig. 9, the cluster lifetime was increased in comparison to the EVAC-AV algorithm when we applied different $D_{t h} s$. The average cluster lifetime was (51 sec) while the average cluster lifetime of EVAC-AV is (18.84 sec) at $D_{t h}=50 \mathrm{~m}$ during the simulation time. At $D_{t h}=70 \mathrm{~m}$, the cluster lifetime of our algorithm is $(50.57 \mathrm{sec})$ whereas, the EVAC_AV is (26.52 $\mathrm{sec})$. Also, the number of clusters has been more than halved compared to the EVAC-AV algorithm at different $D_{t h} S$, as shown in Fig. 10.

Regarding the vehicle's status change, it was measured by calculating the rate of total status changes of the vehicles and applying this step by using a different $D_{t h} s$. The results have been compared with EVAC-AV under the same condition. As displayed in Fig. 11 and Fig. 12, our proposed algorithm shows better results by decreasing the rate of vehicle status change.

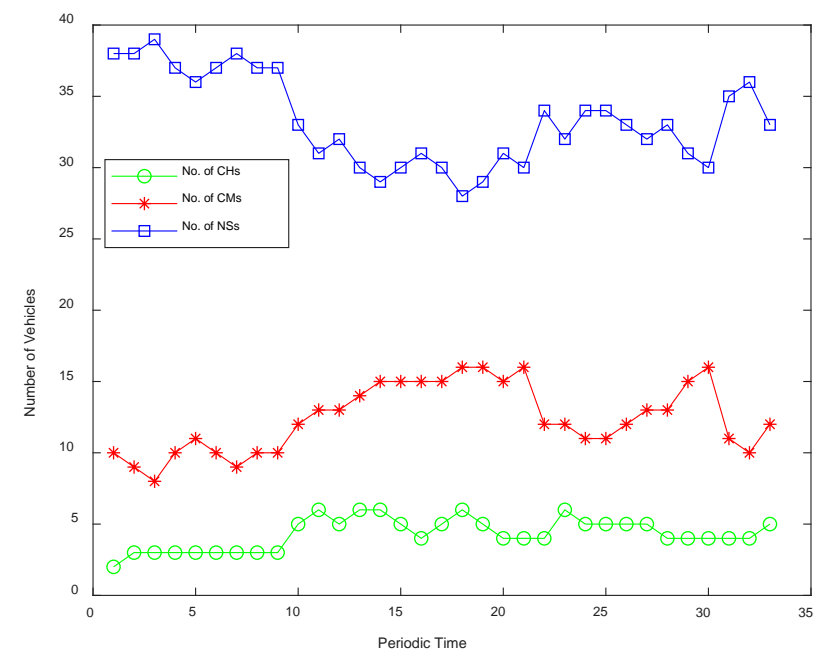

Fig. 6. Vehicles States at $D_{t h}=50 \mathrm{~m}$ 


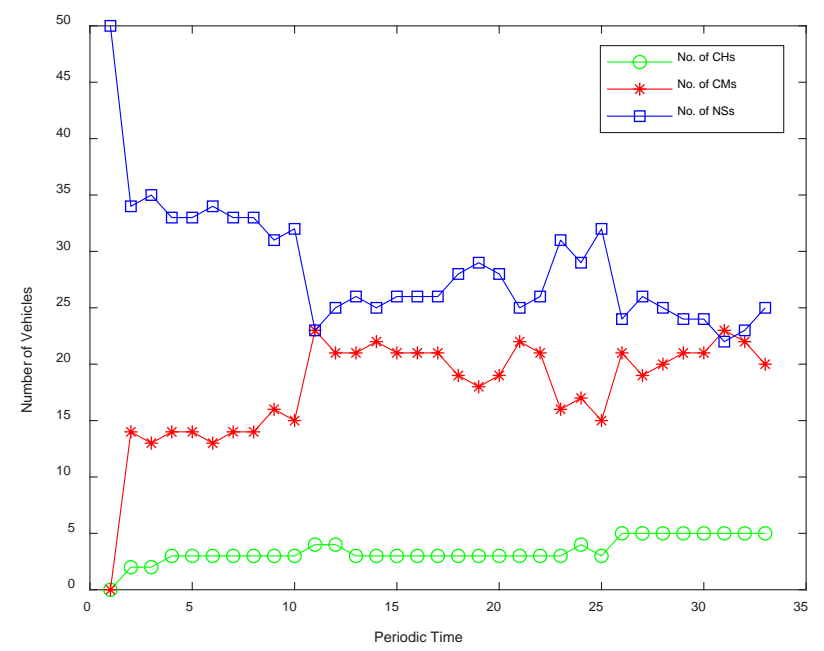

Fig. 7. Vehicles States at $D_{t h}=70 \mathrm{~m}$.
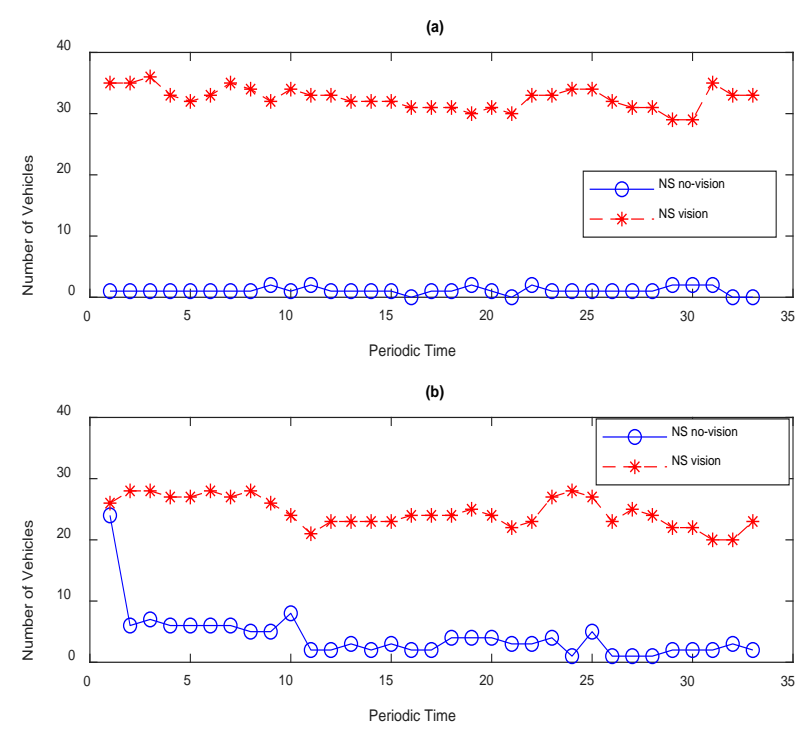

Fig. 8. Distribution of NSs Vehicles According to Vision Area.

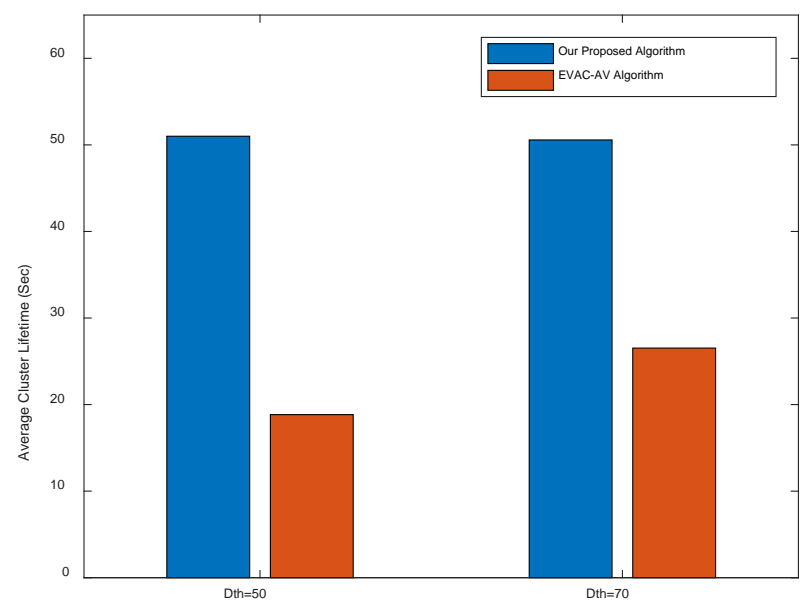

Fig. 9. Average Cluster Lifetime at different $D_{t h} \mathrm{~s}$.

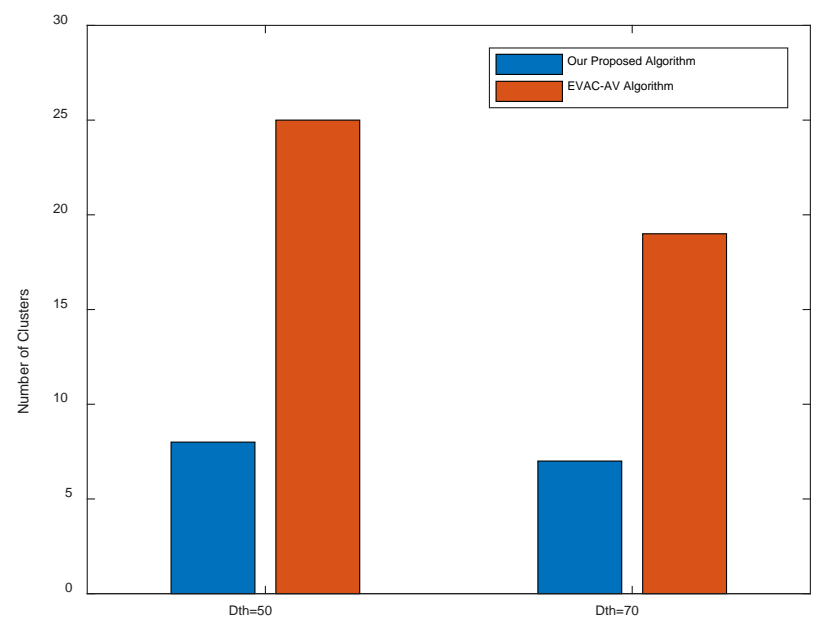

Fig. 10. Number of Clusters at different $D_{t h} \mathrm{~s}$.

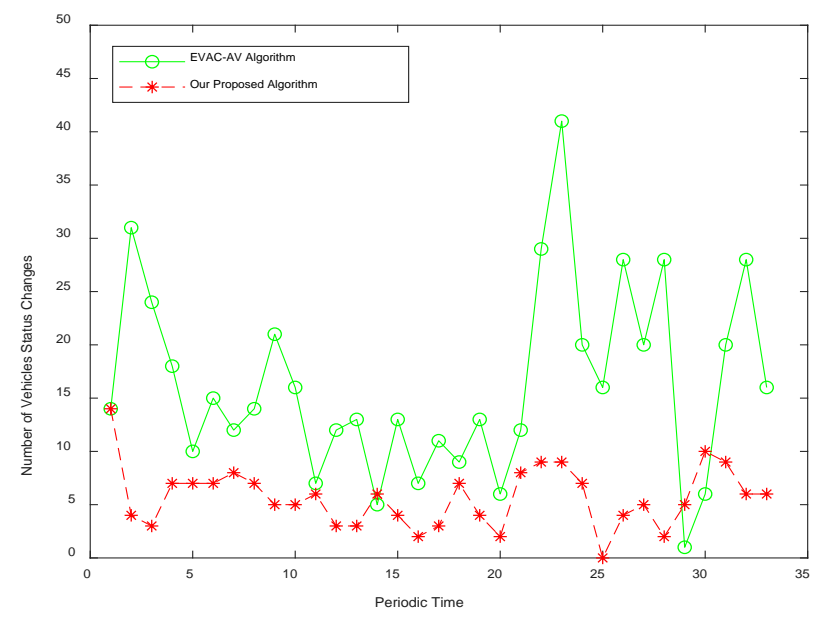

Fig. 11. Vehicles Status Change at $D_{t h}=50 \mathrm{~m}$.

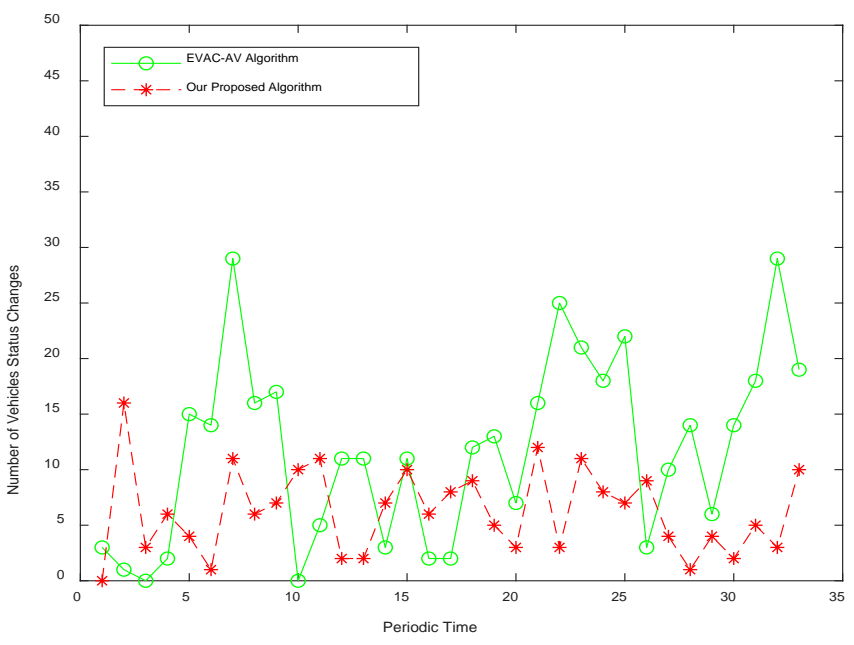

Fig. 12. Vehicles Status Change at $D_{t h}=70 \mathrm{~m}$. 


\section{CONCLUSION}

Video streaming enriches drivers with substantial information for safety, emergency, and entertainment. Clustering algorithms can be used as effective methods to improve and organize the work of the network. In this paper, a new clustering algorithm is proposed for road surveillance. It is characterized not only by the ability to detect and form the clusters when the density increases on the highway but also by finding the optimal $\mathrm{CH}$ for each cluster. The merits of this algorithm come from merging DBSCAN with FLC. DBSCAN algorithm is responsible for forming the cluster when it is triggered by a vehicle need video streaming to know its road condition. The $\mathrm{CH}$ is responsible for providing live road surveillance to all vehicles in the cluster. Fuzzy logic control is used to select the $\mathrm{CH}$ based on the metrics, which are cluster speed, acceleration, and leadership degree. Our proposed algorithm uses the vision area as a crucial parameter, so no vehicle can be nominated to be a $\mathrm{CH}$ if it does not have a sufficient vision area. Simulation results showed that our proposed algorithm provides a lower number of $\mathrm{CHs}$ and clusters and less variation of vehicle status. Additionally, our proposed algorithm increases the cluster lifetime in comparison with the EVAC-AV algorithm, which means the road surveillance service will be more efficient and more stable. In future work, we would like to add some additional metrics like link connectivity duration.

\section{REFERENCES}

[1] S. Al-Sultan, M. M. Al-Doori, A. H. Al-Bayatti, and H. Zedan, "A comprehensive survey on vehicular Ad Hoc network," J. Netw. Comput. Appl., vol. 37, no. 1, pp. 380-392, 2014.

[2] A. Tomatis, H. Menouar, and K. Roscher, "Forwarding in VANETs: GeoNetworking," in Vehicular ad hoc Networks: Standards, Solutions and Research, R. Scopigno, C. Campolo, and A. Molinaro, Eds. Springer, 2015, pp. 221-251.

[3] R. Ghebleh, "A comparative classification of information dissemination approaches in vehicular ad hoc networks from distinctive viewpoints: A survey," Comput. Networks, vol. 131, pp. 15-37, 2018.

[4] K. S. Eunice and I. Juvanna, "Secured Multi-Hop Clustering Protocol for Location-based Routing in VANETs," Int. J. Adv. Comput. Sci. Appl., vol. 10, no. 4, pp. 121-126, 2019.

[5] A. Alharbi, "Survey on Homomorphic Encryption and Address of New Trend," Int. J. Adv. Comput. Sci. Appl., vol. 11, no. 7, pp. 618-626, 2020.

[6] I. T. Abdel-Halim and H. M. A. Fahmy, "Prediction-based protocols for vehicular Ad Hoc Networks: Survey and taxonomy," Comput. Networks, vol. 130, pp. 34-50, 2018.

[7] F. Cunha et al., "Data communication in VANETs: Protocols, applications, and challenges," Ad Hoc Networks, vol. 44, pp. 90-103, 2016.

[8] P. Y. Lai, C. R. Dow, and Y. Y. Chang, "Rapid-Response Framework for Defensive Driving Based on Internet of Vehicles Using MessageOriented Middleware," IEEE Access, vol. 6, pp. 18548-18560, 2018.

[9] T. Phakathi, F. Lugayizi, B. Isong, and N. Gasela, "Quality of Service of
Video Streaming in Vehicular Adhoc Networks: Performance Analysis," Proc. - 2016 Int. Conf. Comput. Sci. Comput. Intell. CSCI 2016, pp. 886-891, 2017.

[10] R. An, Z. Liu, and Y. Ji, "SVC-based cooperative video streaming in highway vehicular networks," Proc. - 31st IEEE Int. Conf. Adv. Inf. Netw. Appl. Work. WAINA 2017, pp. 216-221, 2017.

[11] L. Cai, H. He, L. Sun, A. Huang, and H. Shan, "Channel Allocation for Adaptive Video Streaming in Vehicular Networks," IEEE Trans. Veh. Technol., vol. 66, no. 1, pp. 1-1, 2016.

[12] E. B. Smida, S. G. Fantar, and H. Youssef, "Video streaming challenges over vehicular ad-hoc networks in smart cities," 2017 Int. Conf. Smart, Monit. Control. Cities, SM2C 2017, pp. 12-16, 2017.

[13] C. Cooper, D. Franklin, M. Ros, F. Safaei, and M. Abolhasan, "A Comparative Survey of VANET Clustering Techniques," IEEE Commun. Surv. Tutorials, vol. 19, no. 1, pp. 657-681, 2017.

[14] A. H. Abbas, M. I. Habelalmateen, L. Audah, and N. A. M. Alduais, "A Novel Intelligent Cluster-Head ( $\mathrm{ICH}$ ) to Mitigate the Handover Problem of Clustering in VANETs," Int. J. Adv. Comput. Sci. Appl., vol. 10, no. 6, pp. 194-203, 2019.

[15] W. Qi, Q. Song, X. Wang, and Z. Ning, "SDN-Enabled Social-Aware Clustering in 5G-VANET Systems," IEEE Access, vol. 6, 2018.

[16] B. Hassanabadi, C. Shea, L. Zhang, and S. Valaee, "Clustering in Vehicular Ad Hoc Networks using Affinity Propagation," Ad Hoc Networks, vol. 13, no. PART B, pp. 535-548, 2014.

[17] F. Yang, Z. Lin, and Y. Tang, "A Traffic Flow Based Clustering Scheme for VANETs," Sensors \& Transducers, vol. 180, no. 10, pp. 110-116, 2014.

[18] A. A. Khan, M. Abolhasan, and W. Ni, "An evolutionary game theoretic approach for stable and optimized clustering in vanets," IEEE Trans. Veh. Technol., vol. 67, no. 5, pp. 4501-4513, 2018.

[19] A. Mehmood et al., "ANTSC: An Intelligent Naïve Bayesian Probabilistic Estimation Practice for Traffic Flow to Form Stable Clustering in VANET," IEEE Access, vol. 6, pp. 4452-4461, 2017.

[20] A. ÇALHAN, "A fuzzy logic based clustering strategy for improving vehicular ad-hoc network performance," Proc. Eng. Sci., vol. 40, no. 2, pp. 351-367, 2015.

[21] K. Ozera, K. Bylykbashi, Y. Liu, and L. Barolli, "A fuzzy-based approach for cluster management in VANETs: Performance evaluation for two fuzzy-based systems," Internet of Things, vol. 3-4, pp. 120-133, 2018.

[22] M. S. Talib, A. Hassan, B. Hussin, Z. A. Abas, Z. Saad, and Z. Sabah, "A Novel Stable Clustering Approach based on Gaussian Distribution and Relative Velocity in VANETs," Int. J. Adv. Comput. Sci. Appl., vol. 9, no. 4, pp. 216-220, 2018.

[23] C. M. Huang, H. L. Wang, H. Zhou, S. Xu, and D. Ren, "EVAC-AV: The live road surveillance control scheme using an effective-vision-areabased clustering algorithm with the adaptive video-streaming technique," IEEE Syst. J., vol. 11, no. 3, pp. 1228-1238, 2017.

[24] M. Ester, "Density-Based Clustering," in Data Clustering Algorithms and Applications, Minnesota: Crc, Hall, 2014, pp. 111-145.

[25] M. Ester, H.-P. Kriegel, J. Sander, and X. Xu, "A density-based algorithm for discovering clusters in large spatial databases with noise.," in Kdd, 1996, vol. 96, no. 34, pp. 226-231.

[26] R. A. Shaikh, "Fuzzy Risk-based Decision Method for Vehicular Ad Hoc Networks," Int. J. Adv. Comput. Sci. Appl., vol. 7, no. 9, pp. 54-62, 2016. 